This is an electronic reprint of the original article. This reprint may differ from the original in pagination and typographic detail.

Author(s): Ruuska, Sauli; Miettinen, Kaisa; Wiecek, Margaret M.

Title: Connections between single-level and bilevel multiobjective optimization

Year: $\quad 2012$

Version:

Please cite the original version:

Ruuska, S., Miettinen, K., \& Wiecek, M. M. (2012). Connections between single-level and bilevel multiobjective optimization. Journal of Optimization Theory and Applications, 153(1), 60-74. https://doi.org/10.1007/s10957-011-9943-y

All material supplied via JYX is protected by copyright and other intellectual property rights, and duplication or sale of all or part of any of the repository collections is not permitted, except that material may be duplicated by you for your research use or educational purposes in electronic or print form. You must obtain permission for any other use. Electronic or print copies may not be offered, whether for sale or otherwise to anyone who is not an authorised user. 


\title{
Connections Between Single-Level and Bilevel Multiobjective Optimization
}

\author{
Sauli Ruuska - Kaisa Miettinen . \\ Margaret M. Wiecek
}

\begin{abstract}
The relationship between bilevel optimization and multiobjective optimization has been studied by several authors and there have been repeated attempts to establish a link between the two. We unify the results from the literature and generalize them for bilevel multiobjective optimization. We formulate sufficient conditions for an arbitrary binary relation to guarantee equality between the efficient set produced by the relation and the set of optimal solutions to a bilevel problem. In addition, we present specially structured bilevel multiobjective optimization problems motivated by real-life applications and an accompanying binary relation permitting their reduction to single-level multiobjective optimization problems.
\end{abstract}

Keywords Two-level optimization $\cdot$ Multiobjective programming $\cdot$ Multicriteria optimization - Binary relations

\section{Introduction}

A bilevel optimization problem consists of two coupled optimization problems with two decision makers, the leader and the follower. The leader's problem, or the upper-level problem, is to make an optimal decision in anticipation of the follower's response. The follower's problem, or the lower-level problem, is to make an optimal decision given the leader's deci-

This research was supported in part by Forestcluster Ltd under research program Intelligent, resource-efficient production technologies (EffTech); Doctoral Programme in Systems Analysis, Decision Making and Risk Management; and Jenny and Antti Wihuri Foundation, Finland.

S. Ruuska (corresponding author)

Department of Mathematical Information Technology, University of Jyväskylä, Jyväskylä, FINLAND

E-mail: sauli.ruuska@jyu.fi

K. Miettinen

Department of Mathematical Information Technology, University of Jyväskylä, Jyväskylä, FINLAND

E-mail: kaisa.miettinen@jyu.fi

M. M. Wiecek

Department of Mathematical Information Technology, University of Jyväskylä, Jyväskylä, FINLAND

On sabbatical leave from Department of Mathematical Sciences, Clemson University, Clemson, SC, U.S.A.

E-mail:wmalgor@clemson.edu 
sion. Thus, the lower-level problem is parameterized by the upper-level decision and appears as a constraint in the upper-level problem.

Bilevel single-objective optimization has been studied in different formulations and in the context of different applications for more than three decades. The applications of bilevel optimization cover decision making in, for example, organizational hierarchies in which one decision maker is in a subordinate role to another. Bilevel optimization also has applications in various design tasks. Due to the inherent difficulty of bilevel optimization problems, their numerical solution still remains a challenge today. For further background, see the surveys [1-3], and the monographs by Bard [4] and Dempe [5].

There have been several attempts to establish a connection between bilevel single-objective optimization and multiobjective optimization. For the early attempts and a proof that such attempts were in vain, see [6] and the references therein. Fülöp [7] discovered that the feasible set of a bilevel linear program can be represented as the efficient set of an appropriately constructed multiobjective linear program. Consequently, a bilevel optimization problem can be posed as optimization over the efficient set. Fülöp's theorem was popularized by Dempe [5]. It has since been used as a part of an algorithm for solving bilevel linear programs [8], but also criticized for the high number of objectives in the resulting multiobjective problem [9].

Fülöp's theorem was proposed and formulated for bilevel linear programs. Linearity, however, is not necessary for the result. If the lower-level problem is a linear program, then the reformulation results in optimization over the efficient set of a multiobjective linear program. Otherwise, the resulting problem is nonlinear, but the theorem remains valid if only formulated accordingly. In this paper, we formulate Fülöp's theorem for a general vector-valued function, thus allowing the lower-level problem to be a nonlinear multiobjective optimization problem.

Fliege and Vicente [10] proposed a binary relation such that a solution to a bilevel optimization problem is optimal if and only if it is efficient with respect to the proposed relation. The result is stronger than Fülöp's theorem and allows a reformulation of a bilevel optimization problem as a multiobjective optimization problem. An essentially identical binary relation and problem reformulation were later considered by Ivanenko and Plyasunov [11] with the distinction that, compared to the relation of Fliege and Vicente, the relation of Ivanenko and Plyasunov involves an extension allowing the upper-level constraint functions to depend on the lower-level decision.

The theorems of Fülöp and of Fliege and Vicente are closely related. The binary relation proposed by Fliege and Vicente consists of a union of two ordering relations: one concerned with feasibility, and the other with optimality with respect to the upper-level objective. The former can be used to formulate a theorem equivalent to Fülöp's theorem (see [11, Proposition 1]). Conversely, the ordering relation implied by Fülöp's theorem can be used in the union as the relation concerned with feasibility. In this paper, we generalize the binary relation proposed by Fliege and Vicente for bilevel multiobjective optimization and formalize the connection to Fülöp's theorem.

The binary relation proposed by Fliege and Vicente is difficult to evaluate because whether any two decisions are in the relation depends on their optimality to the lower-level problem. The relation can be simplified for certain classes of problems using appropriate optimality conditions [10,11]. In [12], the results from [10] were reiterated, and it was suggested that the optimal solutions to a bilevel optimization problem could be obtained as the intersection of two efficient sets: one with respect to the relation concerned with feasibility, and the other with respect to the relation concerned with optimality at the upper level. However, it was not elaborated how such an intersection could be calculated in practice. 
In the last few years, there has been a growing interest in solving bilevel multiobjective optimization problems and the number of methods proposed for solving such problems has increased rapidly. Calvete and Galé [13] and Alves et al. [9] proposed scalarization approaches to bilevel linear programs with multiple objectives at the upper level. Nie [14] and Bonnel and Morgan [15] studied bilevel optimization problems with multiple objectives at the lower level. Nishizaki and Sakawa [16, 17], Dell'Aere [18], and Arora and Arora [19] proposed methods for solving bilevel multiobjective optimization problems by scalarization and substitution of optimality conditions for the lower-level problem.

For nonlinear bilevel multiobjective optimization problems, Eichfelder [20] and Gebhardt and Jahn [21] proposed exact iterative methods. In Eichfelder's method, the solution set is constructed by means of a sequence of iteratively refined approximations of the feasible set, whereas Gebhardt and Jahn propose a method based on a so-called subdivision technique and iterative refinement. Both methods rely on explicit discretization of the set of feasible leader's decisions. Moreover, Shi and Xia [22,23] and Abo-Sinna and Baky [24] proposed interactive methods, Osman et al. [25], Zhang et al. [26], Yano and Sakawa [27], and Baky [28,29] proposed fuzzy approaches, and Yin [30], Deb and Sinha [31], and Jia and Wang [32] proposed evolutionary methods for solving bilevel multiobjective problems.

Introducing multiple objectives to a bilevel problem poses not only technical challenges, but conceptual as well. With multiple objectives at the lower level, one can no longer assume that there exists a unique solution to the lower-level problem. The different formulations resulting from the leader's anticipations of the follower's actions were analyzed by Nishizaki and Sakawa [16] and Nie [14]. A common approach is to assume that if the set of optimal solutions to the lower-level problem is not a singleton, then the upper-level decision maker is allowed to make the choice. This assumption brings about what is known as the optimistic bilevel optimization problem, whereas the opposite case, in which the lower-level decision is always the one least preferred by the upper-level decision maker, is known as the pessimistic formulation $[5,16]$.

To our knowledge, the only result found in the literature that shows a connection between bilevel multiobjective optimization and multiobjective optimization is a theorem due to Eichfelder [20]. Eichfelder's theorem states that the feasible set of a bilevel multiobjective optimization problem can be represented as the efficient set of a multiobjective optimization problem. It is based on an ordering relation similar to the part concerning feasibility in the relation proposed by Fliege and Vicente, and it is roughly equivalent to the multiobjective generalization of Fülöp's theorem discussed above.

In this paper, we study the relationship between bilevel optimization and multiobjective optimization. In particular, we unify some existing results that have been independently developed by different authors and generalize them for bilevel multiobjective optimization. In addition, we give sufficient conditions for an arbitrary binary relation to guarantee equality between the efficient set produced by the relation and the set of optimal solutions to a bilevel problem. The binary relations satisfying the conditions can be used to reformulate bilevel multiobjective problems as multiobjective optimization problems; in effect, the original problem is reduced to a single-level problem. Motivated by real-life applications, we present specially structured bilevel multiobjective problems that can benefit from this reduction.

The paper is structured as follows. Problem formulation and necessary definitions are given in Section 2. Results relating bilevel optimization and multiobjective optimization are presented in Section 3. We lay a basis for deriving new binary relations connecting bilevel and multiobjective optimization in Section 4, and propose a simple binary relation that enables the reduction of bilevel multiobjective problems to single-level problems in certain 
special cases. The special cases are introduced in Section 5 and the paper is concluded in Section 6.

\section{Problem Formulation}

Let $X \subset \mathbb{R}^{n}$ be a nonempty set, and let $f: X \rightarrow \mathbb{R}^{p}$ be a vector-valued function. A general multiobjective optimization problem (MOP) has the form

$$
\min f(\mathbf{x}) \text { subject to } \mathbf{x} \in X,
$$

where $X$ is the feasible set and $f$ is the objective function. Vector $\mathbf{x} \in X, \mathbf{x}=\left(x_{1}, \ldots, x_{n}\right)$, is called a (feasible) decision, and its image $\mathbf{y}=f(\mathbf{x}), \mathbf{y}=\left(y_{1}, \ldots, y_{p}\right)$, an outcome.

The minimization in (1) is understood as finding the set of decisions corresponding to the set of minimal outcomes in the set $Y:=\{f(\mathbf{x}): \mathbf{x} \in X\}$ with respect to a given binary relation $\preccurlyeq$ on $Y$. For $\mathbf{y}, \overline{\mathbf{y}} \in Y$, if $\overline{\mathbf{y}} \preccurlyeq \mathbf{y}$, then $\overline{\mathbf{y}}$ is said to dominate $\mathbf{y}$ with respect to $\preccurlyeq$. If there does not exist an outcome $\overline{\mathbf{y}} \in Y$ such that $\overline{\mathbf{y}} \preccurlyeq \mathbf{y}$, then $\mathbf{y}$ is said to be nondominated in $Y$. A decision $\mathbf{x} \in X$ is said to be efficient if its image under $f$ is nondominated. The set of nondominated outcomes is denoted by $N(Y, \preccurlyeq)$ and called the nondominated set. The set of efficient decisions is denoted by $E(X, f, \preccurlyeq)$ and called the efficient set.

Certain properties are often assumed for the relation $\preccurlyeq$ to make sure that problem (1) has a meaningful solution. For example, it is common to consider only binary relations compatible with addition and positive scalar multiplication, which can be represented as cones in $\mathbb{R}^{p}$ (see, e.g., $[33,34]$ ). In particular, the following (partial) ordering relations are ubiquitous in the field of multiobjective optimization:

$$
\begin{aligned}
& \overline{\mathbf{y}}<\mathbf{y} \stackrel{\text { def }}{\Longleftrightarrow} \bar{y}_{i}<y_{i} \text { for all } i=1, \ldots, p, \\
& \overline{\mathbf{y}} \leq \mathbf{y} \stackrel{\text { def }}{\Longleftrightarrow} \bar{y}_{i} \leq y_{i} \text { for all } i=1, \ldots, p \text { and } \mathbf{y} \neq \overline{\mathbf{y}}, \\
& \overline{\mathbf{y}} \leqq \mathbf{y} \stackrel{\text { def }}{\Longleftrightarrow} \bar{y}_{i} \leq y_{i} \text { for all } i=1, \ldots, p .
\end{aligned}
$$

Nondominated outcomes and efficient decisions with respect to the relations $\leq$ and $<$ are called Pareto optimal and weakly Pareto optimal, respectively (see, e.g., [35]). Unless otherwise stated, the minimization in (1) is defined with respect to the relation $\leq$.

Let $X_{u} \subset \mathbb{R}^{n_{u}}$ and $X_{l}\left(\mathbf{x}_{u}\right) \subset \mathbb{R}^{n_{l}}$ for all $\mathbf{x}_{u} \in X_{u}$ be nonempty sets, and let

$$
X=\left\{\left(\mathbf{x}_{u}, \mathbf{x}_{l}\right): \mathbf{x}_{u} \in X_{u}, \mathbf{x}_{l} \in X_{l}\left(\mathbf{x}_{u}\right)\right\} \subset \mathbb{R}^{n},
$$

where $n=n_{u}+n_{l}$. An optimistic bilevel multiobjective optimization problem (BMOP) has the form

$$
\begin{gathered}
\min _{\mathbf{x}_{u}, \mathbf{x}_{l}} f_{u}\left(\mathbf{x}_{u}, \mathbf{x}_{l}\right) \\
\text { subject to } \mathbf{x}_{l} \in E\left(X_{l}\left(\mathbf{x}_{u}\right), f_{l}\left(\mathbf{x}_{u}, \cdot\right), \leq\right), \\
\mathbf{x}_{u} \in X_{u},
\end{gathered}
$$

where $f_{u}: X \rightarrow \mathbb{R}^{p_{u}}$ is the upper-level objective function and $f_{l}: X \rightarrow \mathbb{R}^{p_{l}}$ is the lower-level objective function. Vector $\mathbf{x}_{u}$ is called the upper-level decision and vector $\mathbf{x}_{l}$ the lower-level decision. In the rest of this paper, a shorthand notation $\mathbf{x}=\left(\mathbf{x}_{u}, \mathbf{x}_{l}\right)$ is frequently used to improve readability.

The notation used for the set $X_{l}$ reflects the fact that, in general, the feasible set of the lower-level problem depends on the upper-level decision $\mathbf{x}_{u}$. Some authors consider a more 
general problem where, in addition, the set $X_{u}$ may depend on the lower-level decision $\mathbf{x}_{l}$. Such problems are, however, beyond the scope of this paper.

The feasible set of (2), denoted here by $X_{\mathrm{I}}$,

$$
X_{\mathrm{I}}:=\left\{\mathbf{x}: \mathbf{x}_{u} \in X_{u}, \mathbf{x}_{l} \in E\left(X_{l}\left(\mathbf{x}_{u}\right), f_{l}\left(\mathbf{x}_{u}, \cdot\right), \leq\right)\right\},
$$

is called the induced set of (2) and consists of all the decisions $\mathbf{x} \in X$ such that $\mathbf{x}_{l}$ is efficient to the lower-level problem with an upper-level decision $\mathbf{x}_{u}$. We assume minimization with respect to the relation $\leq$ at both the upper and the lower level of problem (2). The efficient set of (2) is then, by definition, $E\left(X_{\mathrm{I}}, f_{u}, \leq\right)$. Thus, the induced set allows problem (2) to be written compactly as

$$
\min f_{u}(\mathbf{x}) \text { subject to } \mathbf{x} \in X_{\mathrm{I}} \text {. }
$$

From problem (4), it is evident that the lower-level problem only appears as a constraint in the upper-level problem.

If the functions $f_{u}$ and $f_{l}$ are scalar valued, then problem (2) reduces to an ordinary bilevel optimization problem, which can be written as

$$
\begin{aligned}
\min _{\mathbf{x}} & f_{u}\left(\mathbf{x}_{u}, \mathbf{x}_{l}\right) \\
\text { subject to } \mathbf{x}_{l} & =\arg \min _{\overline{\mathbf{x}}_{l}}\left\{f_{l}\left(\mathbf{x}_{u}, \overline{\mathbf{x}}_{l}\right): \overline{\mathbf{x}}_{l} \in X_{l}\left(\mathbf{x}_{u}\right)\right\}, \\
\mathbf{x}_{u} & \in X_{u}
\end{aligned}
$$

assuming that for all $\mathbf{x}_{u} \in X_{u}$ there exists a unique optimal solution $\mathbf{x}_{l}\left(\mathbf{x}_{u}\right)$ to the lower-level problem. The induced set of (5) is

$$
\left\{\mathbf{x}: \mathbf{x}_{u} \in X_{u}, \mathbf{x}_{l}=\arg \min _{\overline{\mathbf{x}}_{l}}\left\{f_{l}\left(\mathbf{x}_{u}, \overline{\mathbf{x}}_{l}\right): \overline{\mathbf{x}}_{l} \in X_{l}\left(\mathbf{x}_{u}\right)\right\}\right\}
$$

which is clearly a special case of (3). Problem (5) also has a compact formulation analogous to (4), but using (6) instead of (3).

By a theorem due to Fülöp [7], the induced set of (5) can, under certain assumptions, be represented as an efficient set of a MOP. It follows that problem (5) is equivalent to a problem of the form

$$
\min f_{u}(\mathbf{x}) \text { subject to } \mathbf{x} \in E(X, \bar{f}, \leq),
$$

where $\bar{f}$ is some vector-valued function defined on $X$. Problem (7) is known as optimization over the efficient set and it has been studied especially in the context of multiobjective linear programs (see, e.g., [36-41]). We present Fülöp's theorem generalized for problem (2) in the next section.

\section{Representations by Efficient Sets}

In this section, we review the results from the literature that establish a connection between bilevel optimization and multiobjective optimization. We also generalize the theorems that were proposed for single-objective bilevel optimization to bilevel multiobjective optimization with vector-valued objective functions allowed at both levels. What is common to the results presented in this section is that they allow a reformulation of a bilevel optimization problem by representing the problem or some aspect of it as the efficient set of a specially crafted MOP.

We begin by presenting theorems relating the induced set of a bilevel problem to the efficient set of a multiobjective problem. We then present a theorem connecting the solution 
set of a bilevel problem to the efficient set of a multiobjective problem and conclude the section by formalizing the relationships among the theorems presented. All the theorems are given in a unified form that makes a direct comparison possible.

The first theorem states that the induced set $X_{\text {I }}$ can be represented as the efficient set of a MOP. It is a generalization of a theorem given by Fülöp [7, Proposition 2.1] for bilevel single-objective linear programs. For the generalized theorem, we adopt the concise formulation of Fülöp's theorem given in [8].

Let $\mathbf{e} \in \mathbb{R}^{n_{u}}$ be a vector with each component equal to 1 , and let $\bar{f}_{\mathrm{F}}$ be the function $\bar{f}_{\mathrm{F}}: X \rightarrow \mathbb{R}^{n_{u}+1+p_{l}}: \mathbf{x} \rightarrow\left(\mathbf{x}_{u},\left\langle\mathbf{e},-\mathbf{x}_{u}\right\rangle, f_{l}(\mathbf{x})\right)$, where $\langle\cdot, \cdot\rangle$ denotes the scalar product in $\mathbb{R}^{n_{u}}$.

Theorem 3.1 The efficient set $E\left(X, \bar{f}_{\mathrm{F}}, \leq\right)$ is equal to the induced set $X_{\mathrm{I}}$ of problem (2).

Proof

1. Let $\mathbf{x} \in E\left(X, \bar{f}_{\mathrm{F}}, \leq\right)$ and assume, to the contrary, that $\mathbf{x} \notin X_{\mathrm{I}}$. Then there exists $\overline{\mathbf{x}}_{l} \in X_{l}\left(\mathbf{x}_{u}\right)$ such that $f_{l}\left(\mathbf{x}_{u}, \overline{\mathbf{x}}_{l}\right) \leq f_{l}(\mathbf{x})$. Let $\hat{\mathbf{x}}$ equal the vector $\left(\mathbf{x}_{u}, \overline{\mathbf{x}}_{l}\right)$. It follows that $\hat{\mathbf{x}} \in X$ and $\bar{f}_{\mathrm{F}}(\hat{\mathbf{x}}) \leq \bar{f}_{\mathrm{F}}(\mathbf{x})$, which is a contradiction. Hence, $\mathbf{x} \in X_{\mathrm{I}}$.

2. Let $\mathbf{x} \in X_{\mathrm{I}}$ and assume, to the contrary, that $\mathbf{x} \notin E\left(X, \bar{f}_{\mathrm{F}}, \leq\right)$. Then there exists $\overline{\mathbf{x}} \in X$ such that $\bar{f}_{\mathrm{F}}(\overline{\mathbf{x}}) \leq \bar{f}_{\mathrm{F}}(\mathbf{x})$. By definition, $\overline{\mathbf{x}}_{u} \leqq \mathbf{x}_{u},\left\langle\mathbf{e},-\overline{\mathbf{x}}_{u}\right\rangle \leq\left\langle\mathbf{e},-\mathbf{x}_{u}\right\rangle, f_{l}(\overline{\mathbf{x}}) \leqq f_{l}(\mathbf{x})$, and $\left(\overline{\mathbf{x}}_{u},\left\langle\mathbf{e},-\overline{\mathbf{x}}_{u}\right\rangle, f_{l}(\overline{\mathbf{x}})\right) \neq\left(\mathbf{x}_{u},\left\langle\mathbf{e},-\mathbf{x}_{u}\right\rangle, f_{l}(\mathbf{x})\right)$. It necessarily follows that $\overline{\mathbf{x}}_{u}=\mathbf{x}_{u}$ because $\overline{\mathbf{x}}_{u} \leq \mathbf{x}_{u}$ would imply $\left\langle\mathbf{e},-\overline{\mathbf{x}}_{u}\right\rangle>\left\langle\mathbf{e},-\mathbf{x}_{u}\right\rangle$. Therefore, $f_{l}(\overline{\mathbf{x}}) \leq f_{l}(\mathbf{x})$, which is a contradiction. Hence, $\mathbf{x} \in E\left(X, \bar{f}_{\mathrm{F}}, \leq\right)$, which completes the proof.

Independently of Fülöp, a theorem similar to Theorem 3.1 was given by Eichfelder [20] for a nonlinear BMOP. We reproduce it here without a proof.

Let $\bar{f}_{\mathrm{E}}$ be the function $\bar{f}_{\mathrm{E}}: X \rightarrow \mathbb{R}^{n_{u}+p_{l}}: \mathbf{x} \rightarrow\left(\mathbf{x}_{u}, f_{l}(\mathbf{x})\right)$, and let $\preccurlyeq_{\mathrm{E}}$ be a binary relation on $\bar{f}_{\mathrm{E}}(X)$ defined as

$$
\bar{f}_{\mathrm{E}}(\overline{\mathbf{x}}) \preccurlyeq_{\mathrm{E}} \bar{f}_{\mathrm{E}}(\mathbf{x}) \stackrel{\text { def }}{\Longleftrightarrow} \overline{\mathbf{x}}_{u}=\mathbf{x}_{u} \wedge f_{l}(\overline{\mathbf{x}}) \leq f_{l}(\mathbf{x}) .
$$

Theorem 3.2 (Eichfelder [20, Theorem 4.1]) The efficient set $E\left(X, \bar{f}_{\mathrm{E}}, \preccurlyeq \mathrm{E}\right)$ is equal to the induced set $X_{\mathrm{I}}$ of problem (2).

A prominent feature of the MOPs resulting from Theorems 3.1 and 3.2 is that their objective functions involve an identity mapping of the upper-level decision $\mathbf{x}_{u}$, which makes it possible to differentiate between the outcomes corresponding to different leader's decisions. The image space of the function $\bar{f}_{\mathrm{E}}$ in Theorem 3.2 has a dimension one less than the image space of the function $\bar{f}_{\mathrm{F}}$ in Theorem 3.1 because of the specialized dominance relation used in Theorem 3.2. Nonetheless, the reasoning behind the two theorems is identical. Both of the theorems result in MOPs with the number of objectives greater than the dimension of the upper-level decision in the original problem. Therefore, their applicability depends in part on the capability of available solution methods to cope with a high number of objectives.

The following theorem states that not only the induced set, but also the efficient set of (2) can be represented as the efficient set of a MOP. It is a multiobjective generalization of a theorem given by Fliege and Vicente [10, Theorem 4.1].

Let $\bar{f}_{\mathrm{FV}}$ be the function $\bar{f}_{\mathrm{FV}}: X \rightarrow \mathbb{R}^{n_{u}+p_{u}+p_{l}}: \mathbf{x} \rightarrow\left(\mathbf{x}_{u}, f_{u}(\mathbf{x}), f_{l}(\mathbf{x})\right)$, and let $\preccurlyeq_{\mathrm{FV}}$ be a binary relation on $\bar{f}_{\mathrm{FV}}(X)$ defined as

$$
\begin{aligned}
& \bar{f}_{\mathrm{FV}}(\overline{\mathbf{x}}) \preccurlyeq \mathrm{FV} \\
& \bar{f}_{\mathrm{FV}}(\mathbf{x}) \stackrel{\mathrm{def}}{\Longleftrightarrow}\left(\overline{\mathbf{x}}_{u}=\mathbf{x}_{u} \wedge f_{l}(\overline{\mathbf{x}}) \leq f_{l}(\mathbf{x})\right) \vee \\
& \left(\left[\nexists \hat{\mathbf{x}} \in X: \hat{\mathbf{x}}_{u}=\overline{\mathbf{x}}_{u} \wedge f_{l}(\hat{\mathbf{x}}) \leq f_{l}(\overline{\mathbf{x}})\right] \wedge f_{u}(\overline{\mathbf{x}}) \leq f_{u}(\mathbf{x})\right) .
\end{aligned}
$$


Theorem 3.3 The efficient set $E\left(X, \bar{f}_{\mathrm{FV}}, \preccurlyeq_{\mathrm{FV}}\right)$ is equal to the efficient set $E\left(X_{\mathrm{I}}, f_{u}, \leq\right)$ of problem (2).

Proof

1. Let $\mathbf{x} \in E\left(X, \bar{f}_{\mathrm{FV}}, \preccurlyeq_{\mathrm{FV}}\right)$ and assume, to the contrary, that $\mathbf{x} \notin E\left(X_{\mathrm{I}}, f_{u}, \leq\right)$. Then either $\mathbf{x} \notin X_{\mathrm{I}}$ or there exists another decision $\overline{\mathbf{x}} \in X_{\mathrm{I}}$ such that $f_{u}(\overline{\mathbf{x}}) \leq f_{u}(\mathbf{x})$.

(a) If $\mathbf{x} \notin X_{\mathrm{I}}$, then there exists $\overline{\mathbf{x}} \in X$ such that $\overline{\mathbf{x}}_{u}=\mathbf{x}_{u}$ and $f_{l}(\overline{\mathbf{x}}) \leq f_{l}(\mathbf{x})$, which is a contradiction. Hence, $\mathbf{x} \in X_{\mathrm{I}}$.

(b) If there exists $\overline{\mathbf{x}} \in X$ such that $f_{u}(\overline{\mathbf{x}}) \leq f_{u}(\mathbf{x})$ and $\overline{\mathbf{x}} \in X_{\mathrm{I}}$, then there does not exist $\hat{\mathbf{x}} \in X$ for which $\hat{\mathbf{x}}_{u}=\overline{\mathbf{x}}_{u}$ and $f_{l}\left(\hat{\mathbf{x}}_{u}\right) \leq f_{l}\left(\overline{\mathbf{x}}_{u}\right)$, which is a contradiction. Hence, $\overline{\mathbf{x}}$ does not exist.

Therefore, $\mathbf{x} \in E\left(X_{\mathrm{I}}, f_{u}, \leq\right)$.

2. Let $\mathbf{x} \in E\left(X_{\mathrm{I}}, f_{u}, \leq\right)$ and assume, to the contrary, that $\mathbf{x} \notin E\left(X, \bar{f}_{\mathrm{FV}}, \preccurlyeq_{\mathrm{FV}}\right)$. It follows immediately that $\mathbf{x} \in X_{\mathrm{I}} \subset X$, leaving two cases. Let $\overline{\mathbf{x}} \in X$ be a decision such that $\bar{f}_{\mathrm{FV}}(\overline{\mathbf{x}}) \preccurlyeq_{\mathrm{FV}} \bar{f}_{\mathrm{FV}}(\mathbf{x})$. If $\overline{\mathbf{x}}_{u}=\mathbf{x}_{u}$ and $f_{l}(\overline{\mathbf{x}}) \leq f_{l}(\mathbf{x})$, then $\mathbf{x} \notin X_{\mathrm{I}}$, which is a contradiction. Otherwise, if $\overline{\mathbf{x}} \in X_{\mathrm{I}}$ and $f_{u}(\overline{\mathbf{x}}) \leq f_{u}(\mathbf{x})$, then $\mathbf{x} \notin E\left(X_{\mathrm{I}}, f_{u}, \leq\right)$, which is also a contradiction. Hence, $\overline{\mathbf{x}}$ does not exist and $\mathbf{x} \in E\left(X, \bar{f}_{\mathrm{FV}}, \preccurlyeq_{\mathrm{FV}}\right)$, which completes the proof.

If there are no upper-level objectives in (2), or if the upper-level objective function $f_{u}$ is constant, then the induced set and the efficient set of problem (2) are equal and Theorem 3.3 reduces to Theorem 3.2. Theorem 3.2 is hence a special case of Theorem 3.3. While there is no such relationship between Theorems 3.3 and 3.1, it is easy to formulate a theorem similar to Theorem 3.3 but containing Theorem 3.1 as a special case. The following two results describe the connections between Theorem 3.3 and Theorems 3.1 and 3.2.

Proposition 3.1 For all $\mathbf{x}, \overline{\mathbf{x}} \in X$, the statements $\bar{f}_{\mathrm{E}}(\overline{\mathbf{x}}) \preccurlyeq_{\mathrm{E}} \bar{f}_{\mathrm{E}}(\mathbf{x})$ and $\bar{f}_{\mathrm{F}}(\overline{\mathbf{x}}) \leq \bar{f}_{\mathrm{F}}(\mathbf{x})$ are equivalent.

Proof Let $\mathbf{x}, \overline{\mathbf{x}} \in X$. If $\bar{f}_{\mathrm{E}}(\overline{\mathbf{x}}) \preccurlyeq_{\mathrm{E}} \bar{f}_{\mathrm{E}}(\mathbf{x})$, then $\overline{\mathbf{x}}_{u}=\mathbf{x}_{u} \wedge f_{l}(\overline{\mathbf{x}}) \leq f_{l}(\mathbf{x})$ and, hence, $\bar{f}_{\mathrm{F}}(\overline{\mathbf{x}}) \leq \bar{f}_{\mathrm{F}}(\mathbf{x})$. If $\bar{f}_{\mathrm{F}}(\overline{\mathbf{x}}) \leq \bar{f}_{\mathrm{F}}(\mathbf{x})$, then $\overline{\mathbf{x}}_{u} \leqq \mathbf{x}_{u}$ and $\left\langle\mathbf{e},-\overline{\mathbf{x}}_{u}\right\rangle \leq\left\langle\mathbf{e},-\mathbf{x}_{u}\right\rangle$, which implies that $\overline{\mathbf{x}}_{u}=\mathbf{x}_{u} \wedge f_{l}(\overline{\mathbf{x}}) \leq$ $f_{l}(\mathbf{x})$ and, hence, $\bar{f}_{\mathrm{E}}(\overline{\mathbf{x}}) \preccurlyeq \mathrm{E} \bar{f}_{\mathrm{E}}(\mathbf{x})$.

Corollary 3.1 For all $\mathbf{x}, \overline{\mathbf{x}} \in X$, the following statements are equivalent:

(a) $\bar{f}_{\mathrm{FV}}(\overline{\mathbf{x}}) \preccurlyeq \mathrm{FV} \bar{f}_{\mathrm{FV}}(\mathbf{x})$,

(b) $\bar{f}_{\mathrm{E}}(\overline{\mathbf{x}}) \preccurlyeq_{\mathrm{E}} \bar{f}_{\mathrm{E}}(\mathbf{x}) \vee\left(\left[\nexists \hat{\mathbf{x}} \in X: \bar{f}_{\mathrm{E}}(\hat{\mathbf{x}}) \preccurlyeq_{\mathrm{E}} \bar{f}_{\mathrm{E}}(\overline{\mathbf{x}})\right] \wedge f_{u}(\overline{\mathbf{x}}) \leq f_{u}(\mathbf{x})\right)$,

(c) $\bar{f}_{\mathrm{F}}(\overline{\mathbf{x}}) \leq \bar{f}_{\mathrm{F}}(\mathbf{x}) \vee\left(\left[\nexists \hat{\mathbf{x}} \in X: \bar{f}_{\mathrm{F}}(\hat{\mathbf{x}}) \leq \bar{f}_{\mathrm{F}}(\overline{\mathbf{x}})\right] \wedge f_{u}(\overline{\mathbf{x}}) \leq f_{u}(\mathbf{x})\right)$.

Proof The statements (a) and (b) are equivalent by definition. The statements (b) and (c) are equivalent by Proposition 3.1.

By Corollary 3.1, Theorem 3.3 could alternatively have been formulated in accordance with Theorem 3.1 and not Theorem 3.2. However, in Theorem 3.2, the intent of the result is perhaps more apparent from the formulation than in Theorem 3.1. Therefore, we believe that the chosen formulation makes the presentation easier to follow.

The virtue of Theorem 3.3 is that it allows a reduction of problem (2) to a MOP. Unfortunately, the inherent difficulty of bilevel optimization is introduced into the resulting MOP by the relation $\preccurlyeq_{\mathrm{FV}}$, which depends on the efficiency of the lower-level decisions. Thus, for any two decisions $\mathbf{x}, \overline{\mathbf{x}} \in X$, the computational effort of directly evaluating the statement $\bar{f}_{\mathrm{FV}}(\overline{\mathbf{x}}) \preccurlyeq_{\mathrm{FV}} \bar{f}_{\mathrm{FV}}(\mathbf{x})$ is equivalent in the worst case to that of solving an optimization problem. Moreover, as shown in the next section, the relation $\preccurlyeq_{\mathrm{Fv}}$ is nontransitive in general, which makes it difficult to work with in applications. In the next section, we consider replacing the relation $\preccurlyeq_{\mathrm{FV}}$ in Theorem 3.3 by other, potentially more tractable binary relations. 


\section{Alternative Binary Relations}

The binary relation $\preccurlyeq_{\mathrm{Fv}}$ presented in the previous section is complicated by the fact that, in general, no two decisions can be considered in isolation. This is because of the condition requiring that any decision dominating another decision at the upper level must be efficient to the lower-level problem. The condition makes the relation depend not only on the two decisions at hand, but also on a possibly infinite number of other decisions. Therefore, applying Theorem 3.3 to problem (2) would apparently not make the problem any easier to solve. However, by Corollary 3.1, the reduction of problem (2) to a MOP is not unique. Therefore, there may exist binary relations that could be used in place of the relation $\preccurlyeq_{\mathrm{FV}}$ to obtain more useful reductions of problem (2).

In this section, we first formulate sufficient conditions guaranteeing that, given a set of decisions and an appropriate mapping, an arbitrary binary relation produces the same efficient set as the relation $\preccurlyeq_{\mathrm{FV}}$. We then propose a simple binary relation that satisfies the sufficient conditions in certain special cases of problem (2) enabling a potentially very efficient numerical solution.

Consider problem (2) and let $\bar{f}$ be a vector-valued function with domain $X$ and $\preccurlyeq$ be a binary relation on $\bar{f}(X)$.

Theorem 4.1 The efficient set $E(X, \bar{f}, \preccurlyeq)$ is equal to the efficient set $E\left(X, \bar{f}_{\mathrm{Fv}}, \preccurlyeq \mathrm{Fv}\right)$ if the following conditions are satisfied for all $\mathbf{x}, \overline{\mathbf{x}}, \hat{\mathbf{x}} \in X$ :

(a) (inclusivity) $\bar{f}_{\mathrm{FV}}(\overline{\mathbf{x}}) \preccurlyeq \mathrm{FV} \bar{f}_{\mathrm{FV}}(\mathbf{x}) \Longrightarrow \bar{f}(\overline{\mathbf{x}}) \preccurlyeq \bar{f}(\mathbf{x})$,

(b) (compatibility) $\bar{f}(\overline{\mathbf{x}}) \preccurlyeq \bar{f}(\mathbf{x}) \wedge \overline{\mathbf{x}} \in X_{\mathrm{I}} \Longrightarrow \bar{f}_{\mathrm{FV}}(\overline{\mathbf{x}}) \preccurlyeq \mathrm{FV} \bar{f}_{\mathrm{FV}}(\mathbf{x})$,

(c) (transitivity) $\bar{f}(\overline{\mathbf{x}}) \preccurlyeq \bar{f}(\hat{\mathbf{x}}) \wedge \bar{f}(\hat{\mathbf{x}}) \preccurlyeq \bar{f}(\mathbf{x}) \Longrightarrow \bar{f}(\overline{\mathbf{x}}) \preccurlyeq \bar{f}(\mathbf{x})$.

Proof

1. Let $\mathbf{x} \in E(X, \bar{f}, \preccurlyeq)$ and assume, to the contrary, that $\mathbf{x} \notin E\left(X, \bar{f}_{\mathrm{FV}}, \preccurlyeq \mathrm{Fv}\right)$. Then there exists $\overline{\mathbf{x}} \in X$ such that $\bar{f}_{\mathrm{FV}}(\overline{\mathbf{x}}) \preccurlyeq_{\mathrm{FV}} \bar{f}_{\mathrm{FV}}(\mathbf{x})$, and it follows from (a) that $\bar{f}(\overline{\mathbf{x}}) \preccurlyeq \bar{f}(\mathbf{x})$, which is a contradiction. Hence, $\mathbf{x} \in E\left(X, \bar{f}_{\mathrm{FV}}, \preccurlyeq_{\mathrm{FV}}\right)$.

2. Let $\mathbf{x} \in E\left(X, \bar{f}_{\mathrm{FV}}, \preccurlyeq \mathrm{FV}\right)$ and assume, to the contrary, that $\mathbf{x} \notin E(X, \bar{f}, \preccurlyeq)$. Then there exists $\hat{\mathbf{x}} \in X$ such that $\bar{f}(\hat{\mathbf{x}}) \preccurlyeq \bar{f}(\mathbf{x})$. If $\hat{\mathbf{x}} \in X_{\mathrm{I}}$, it follows from (b) that $\bar{f}_{\mathrm{FV}}(\hat{\mathbf{x}}) \preccurlyeq{ }_{\mathrm{FV}} \bar{f}_{\mathrm{FV}}(\mathbf{x})$, which is a contradiction. Otherwise, there exists $\overline{\mathbf{x}} \in X_{\mathrm{I}}$ such that $\overline{\mathbf{x}}_{u}=\hat{\mathbf{x}}_{u}$ and $f_{l}(\overline{\mathbf{x}}) \leq$ $f_{l}(\hat{\mathbf{x}})$. Thus, $\bar{f}_{\mathrm{FV}}(\overline{\mathbf{x}}) \preccurlyeq_{\mathrm{FV}} \bar{f}_{\mathrm{FV}}(\hat{\mathbf{x}})$ and it follows from (a) that $\bar{f}(\overline{\mathbf{x}}) \preccurlyeq \bar{f}(\hat{\mathbf{x}})$, from (c) that $\bar{f}(\overline{\mathbf{x}}) \preccurlyeq \bar{f}(\mathbf{x})$, and from (b) that $\bar{f}_{\mathrm{FV}}(\overline{\mathbf{x}}) \preccurlyeq \mathrm{FV} \bar{f}_{\mathrm{FV}}(\mathbf{x})$, which is a contradiction. Hence, $\mathbf{x} \in$ $E(X, \bar{f}, \preccurlyeq)$, which completes the proof.

It follows immediately that a binary relation satisfying all three conditions of Theorem 4.1 can be used to reduce problem (2) to a MOP.

Corollary 4.1 The efficient set $E(X, \bar{f}, \preccurlyeq)$ is equal to the efficient set $E\left(X_{\mathrm{I}}, f_{u}, \leq\right)$ of problem (2) if the relation $\preccurlyeq$ satisfies the conditions $(a)$, (b), and (c) of Theorem 4.1.

Proof The result follows directly from Theorems 3.3 and 4.1 .

We next introduce a binary relation $\preccurlyeq_{\mathrm{SC}}$ that satisfies two of the conditions of Theorem 4.1. It is defined on the same domain as the relation $\preccurlyeq_{\mathrm{FV}}$ and has a strong resemblance to it. However, unlike the relation $\preccurlyeq_{\mathrm{FV}}$, the relation $\preccurlyeq_{\mathrm{sC}}$ does not depend on any external information: whether any two decisions are in the relation is fully determined by the decisions themselves and their respective outcomes. Consequently, the relation $\preccurlyeq_{\text {sc }}$ would make a good candidate to substitute for the relation $\preccurlyeq_{\mathrm{FV}}$. 
Let $\preccurlyeq_{\mathrm{SC}}$ be a binary relation on $\bar{f}_{\mathrm{FV}}(X)$ defined as

$$
\begin{aligned}
& \bar{f}_{\mathrm{FV}}(\overline{\mathbf{x}}) \preccurlyeq \mathrm{sC} \\
& \bar{f}_{\mathrm{FV}}(\mathbf{x}) \stackrel{\text { def }}{\Longleftrightarrow}\left(\overline{\mathbf{x}}_{u}=\mathbf{x}_{u} \wedge f_{l}(\overline{\mathbf{x}}) \leq f_{l}(\mathbf{x})\right) \vee \\
& \left(\neg\left[\mathbf{x}_{u}=\overline{\mathbf{x}}_{u} \wedge f_{l}(\mathbf{x}) \leq f_{l}(\overline{\mathbf{x}})\right] \wedge f_{u}(\overline{\mathbf{x}}) \leq f_{u}(\mathbf{x})\right) .
\end{aligned}
$$

Proposition 4.1 The relation $\preccurlyeq_{\mathrm{SC}}$ together with $\bar{f}:=\bar{f}_{\mathrm{FV}}$ satisfies the conditions $(a)$ and $(b)$ of Theorem 4.1.

Proof

1. Let $\mathbf{x}, \overline{\mathbf{x}} \in X$ be any two decisions such that $\bar{f}_{\mathrm{FV}}(\overline{\mathbf{x}}) \preccurlyeq_{\mathrm{FV}} \bar{f}_{\mathrm{FV}}(\mathbf{x})$. If $\overline{\mathbf{x}}_{u}=\mathbf{x}_{u}$ and $f_{l}(\overline{\mathbf{x}}) \leq$ $f_{l}(\mathbf{x})$, then $\bar{f}_{\mathrm{FV}}(\overline{\mathbf{x}}) \preccurlyeq_{\mathrm{SC}} \bar{f}_{\mathrm{FV}}(\mathbf{x})$. Otherwise, $f_{u}(\overline{\mathbf{x}}) \leq f_{u}(\mathbf{x})$ and there does not exist $\hat{\mathbf{x}} \in X$ such that $\hat{\mathbf{x}}_{u}=\overline{\mathbf{x}}_{u}$ and $f_{l}(\hat{\mathbf{x}}) \leq f_{l}(\overline{\mathbf{x}})$. Therefore, either $\mathbf{x}_{u} \neq \overline{\mathbf{x}}_{u}$ or $f_{l}(\mathbf{x}) \not \leq f_{l}(\overline{\mathbf{x}})$, and it follows that $\bar{f}_{\mathrm{FV}}(\overline{\mathbf{x}}) \preccurlyeq_{\mathrm{SC}} \bar{f}_{\mathrm{FV}}(\mathbf{x})$. Hence, the relation $\preccurlyeq_{\mathrm{sC}}$ satisfies (a).

2. Let $\mathbf{x} \in X$ and $\overline{\mathbf{x}} \in X_{\mathrm{I}}$ be any two decisions such that $\bar{f}_{\mathrm{FV}}(\overline{\mathbf{x}}) \preccurlyeq \mathrm{sC}, \bar{f}_{\mathrm{FV}}(\mathbf{x})$. If $\overline{\mathbf{x}}_{u}=\mathbf{x}_{u}$ and $f_{l}(\overline{\mathbf{x}}) \leq f_{l}(\mathbf{x})$, then $\bar{f}_{\mathrm{FV}}(\overline{\mathbf{x}}) \preccurlyeq_{\mathrm{FV}} \bar{f}_{\mathrm{FV}}(\mathbf{x})$. Otherwise, $f_{u}(\overline{\mathbf{x}}) \leq f_{u}(\mathbf{x})$ and from $\overline{\mathbf{x}} \in X_{\mathrm{I}}$ it follows that there does not exist $\hat{\mathbf{x}} \in X$ such that $\hat{\mathbf{x}}_{u}=\overline{\mathbf{x}}_{u}$ and $f_{l}(\hat{\mathbf{x}}) \leq f_{l}(\overline{\mathbf{x}})$. Hence, $\bar{f}_{\mathrm{FV}}(\overline{\mathbf{x}}) \preccurlyeq_{\mathrm{FV}} \bar{f}_{\mathrm{FV}}(\mathbf{x})$ and the relation $\preccurlyeq_{\mathrm{SC}}$ satisfies (b), which completes the proof.

The following counterexample shows that neither the relation $\preccurlyeq_{\mathrm{FV}}$ nor the relation $\preccurlyeq_{\mathrm{SC}}$ is transitive in general. Thus, neither relation satisfies the condition (c) of Theorem 4.1. Consider the simple bilevel problem

$$
\begin{aligned}
\min _{\mathbf{x}} x_{u} & -x_{l} \\
\text { subject to } x_{l} & =\arg \min _{\bar{x}_{l}}\left\{\bar{x}_{l}: \bar{x}_{l} \in\left[\frac{1}{2}\left(x_{u}-1\right), 5\right]\right\}, \\
x_{u} & \in[0,5]
\end{aligned}
$$

with a scalar decision and a scalar outcome at both levels. Let $\mathbf{x}=(1,0), \overline{\mathbf{x}}=(3,1)$, and $\hat{\mathbf{x}}=(3,3)$. Using the notation introduced for (2), it is readily seen that $\mathbf{x}, \overline{\mathbf{x}}, \hat{\mathbf{x}} \in X$ and $\mathbf{x}, \overline{\mathbf{x}} \in$ $X_{\mathrm{I}}$. It follows that $\bar{f}_{\mathrm{FV}}(\mathbf{x}) \preccurlyeq_{\mathrm{Fv}} \bar{f}_{\mathrm{FV}}(\overline{\mathbf{x}})$ and $\bar{f}_{\mathrm{FV}}(\overline{\mathbf{x}}) \preccurlyeq_{\mathrm{FV}} \bar{f}_{\mathrm{FV}}(\hat{\mathbf{x}})$, but $\bar{f}_{\mathrm{FV}}(\mathbf{x}) \nprec_{\mathrm{FV}} \bar{f}_{\mathrm{FV}}(\hat{\mathbf{x}})$. Hence, the relation $\preccurlyeq_{\mathrm{FV}}$ is not transitive, which is also the case for the relation $\preccurlyeq_{\mathrm{SC}}$. Furthermore, $\bar{f}_{\mathrm{FV}}(\mathbf{x}) \preccurlyeq_{\mathrm{SC}} \bar{f}_{\mathrm{FV}}(\overline{\mathbf{x}}), \bar{f}_{\mathrm{FV}}(\overline{\mathbf{x}}) \preccurlyeq_{\mathrm{SC}} \bar{f}_{\mathrm{FV}}(\hat{\mathbf{x}})$, and $\bar{f}_{\mathrm{FV}}(\hat{\mathbf{x}}) \preccurlyeq_{\mathrm{SC}} \bar{f}_{\mathrm{FV}}(\mathbf{x})$, which demonstrates that the relation $\preccurlyeq_{\text {sC }}$ not only is not transitive, it is cyclic.

Because of nontransitivity and cyclicity, the relation $\preccurlyeq_{\mathrm{SC}}$ is not a suitable replacement for the relation $\preccurlyeq_{\mathrm{FV}}$ in general. While this conclusion is not surprising given the simple structure of $\preccurlyeq_{\mathrm{sc}}$, the relation is still useful in certain special cases of problem (2). Two such special cases will be introduced in the next section.

Both the relations $\preccurlyeq_{\mathrm{FV}}$ and $\preccurlyeq_{\mathrm{SC}}$ fail to satisfy the sufficient conditions given in Theorem 4.1 because of the lack of transitivity. While transitivity is an essential property of any relation to be used within numerical solution methods, we note that based on the proof of Theorem 4.1, the condition (c) does not need to be satisfied for all $\mathbf{x}, \overline{\mathbf{x}}, \hat{\mathbf{x}} \in X$. Instead, the result holds even if the condition (c) is satisfied only for all $\mathbf{x}, \hat{\mathbf{x}} \in X$ and $\overline{\mathbf{x}} \in X_{\mathrm{I}}$ such that $\overline{\mathbf{x}}_{u}=\hat{\mathbf{x}}_{u}$ and $f_{l}(\overline{\mathbf{x}}) \leq f_{l}(\hat{\mathbf{x}})$. That is, if one is willing to consider also nontransitive relations, the sufficient conditions given in Theorem 4.1 can be relaxed.

It can be seen that the relation $\preccurlyeq_{\mathrm{FV}}$ satisfies the sufficient conditions given in Theorem 4.1 if they are relaxed as above. Let $\mathbf{x}, \overline{\mathbf{x}}, \hat{\mathbf{x}} \in X$ be three decisions such that $\overline{\mathbf{x}}_{u}=\hat{\mathbf{x}}_{u}$ and $f_{l}(\overline{\mathbf{x}}) \leq f_{l}(\hat{\mathbf{x}})$, and let $\bar{f}_{\mathrm{FV}}(\overline{\mathbf{x}}) \preccurlyeq_{\mathrm{FV}} \bar{f}_{\mathrm{FV}}(\hat{\mathbf{x}})$ and $\bar{f}_{\mathrm{FV}}(\hat{\mathbf{x}}) \preccurlyeq_{\mathrm{FV}} \bar{f}_{\mathrm{FV}}(\mathbf{x})$. It follows that $\hat{\mathbf{x}} \notin X_{\mathrm{I}}$ and, consequently, that $\hat{\mathbf{x}}_{u}=\mathbf{x}_{u}$ and $f_{l}(\hat{\mathbf{x}}) \leq f_{l}(\mathbf{x})$. Therefore, $\overline{\mathbf{x}}_{u}=\mathbf{x}_{u}$ and $f_{l}(\overline{\mathbf{x}}) \leq f_{l}(\mathbf{x})$ and, hence, $\bar{f}_{\mathrm{FV}}(\overline{\mathbf{x}}) \preccurlyeq_{\mathrm{FV}} \bar{f}_{\mathrm{FV}}(\mathbf{x})$. In contrast, the relation $\preccurlyeq_{\mathrm{SC}}$ does not satisfy even the relaxed conditions: in the above counterexample, $\bar{f}_{\mathrm{FV}}(\overline{\mathbf{x}}) \preccurlyeq_{\mathrm{SC}} \bar{f}_{\mathrm{FV}}(\hat{\mathbf{x}})$ and $\bar{f}_{\mathrm{FV}}(\hat{\mathbf{x}}) \preccurlyeq_{\mathrm{SC}} \bar{f}_{\mathrm{FV}}(\mathbf{x})$, 
but $\bar{f}_{\mathrm{FV}}(\overline{\mathbf{x}}) \aleph_{\mathrm{sC}} \bar{f}_{\mathrm{FV}}(\mathbf{x})$ despite the fact that $\bar{x}_{u}=\hat{x}_{u}, \bar{x}_{l}<\hat{x}_{l}$, and $\overline{\mathbf{x}} \in X_{\mathrm{I}}$. This is consistent with the fact that the relation $\preccurlyeq_{F V}$ fully captures the structure of problem (2) whereas the relation $\preccurlyeq_{\text {SC }}$ does not.

\section{Special Cases}

In this section, we formulate two special cases of problem (2) and show that in those special cases the relation $\preccurlyeq_{S C}$ is transitive, and thus satisfies all the conditions of Theorem 4.1. Therefore, the special cases can be solved as MOPs using the relation $\preccurlyeq_{\text {sc }}$.

The first special case is a BMOP with such a structure that all the objectives that depend on the lower-level decision $\mathbf{x}_{l}$ either appear only at the lower level or at both the upper and the lower level. In other words, any objective present at the upper level that is not present at the lower level must depend only on the upper-level decision $\mathbf{x}_{u}$.

Let $X \in \mathbb{R}^{n}$ be defined as in (2), and let $f_{a}: X_{u} \rightarrow \mathbb{R}^{p_{a}}, f_{b}: X \rightarrow \mathbb{R}^{p_{b}}$, and $f_{c}: X \rightarrow \mathbb{R}^{p_{c}}$ be three vector-valued functions. The problem described above can be written as

$$
\begin{aligned}
& \min _{\mathbf{x}}\left(f_{a}\left(\mathbf{x}_{u}\right), f_{b}(\mathbf{x})\right) \\
& \text { subject to } \mathbf{x}_{l} \in E\left(X_{l}\left(\mathbf{x}_{u}\right),\left(f_{b}, f_{c}\right)\left(\mathbf{x}_{u}, \cdot\right), \leq\right), \\
& \mathbf{x}_{u} \in X_{u},
\end{aligned}
$$

where any one of the functions $f_{a}, f_{b}$, or $f_{c}$ may be omitted. The upper-level and the lowerlevel objective functions are $f_{u}=\left(f_{a}, f_{b}\right)$ and $f_{l}=\left(f_{b}, f_{c}\right)\left(\mathbf{x}_{u}, \cdot\right)$, respectively.

This special case has been motivated by an integrated design and control problem with multiple objectives and the control problem fully discretized (see, e.g., [42]). The problem is formulated with the design problem at the upper level and the control problem, which has to be parameterized by the design, at the lower level. In this kind of a problem, the upperlevel objectives, such as investment cost, that are directly related to the design aspects are naturally independent of the control variables. On the other hand, the lower-level objectives that are relevant for the design are repeated at the upper level.

The following proposition provides means to solve (12) as a MOP using the binary relation $\preccurlyeq_{\mathrm{sC}}$ from Section 4 .

Proposition 5.1 Let the set $X$ and the functions $f_{u}$ and $f_{l}$ be defined as in (12). The efficient $\operatorname{set} E\left(X, \bar{f}_{\mathrm{FV}}, \preccurlyeq \mathrm{sC}\right)$ is equal to the efficient set $E\left(X_{\mathrm{I}}, f_{u}, \leq\right)$ of problem (12).

Proof By Proposition 4.1 and Corollary 4.1, it is sufficient to show that the relation $\preccurlyeq_{\mathrm{SC}}$ is transitive for problem (12), that is, for all $\mathbf{x}, \overline{\mathbf{x}}, \hat{\mathbf{x}} \in X$, it follows from $\bar{f}_{\mathrm{FV}}(\overline{\mathbf{x}}) \preccurlyeq \mathrm{sC} \bar{f}_{\mathrm{FV}}(\hat{\mathbf{x}})$ and $\bar{f}_{\mathrm{FV}}(\hat{\mathbf{x}}) \preccurlyeq_{\mathrm{SC}} \bar{f}_{\mathrm{FV}}(\mathbf{x})$ that $\bar{f}_{\mathrm{FV}}(\overline{\mathbf{x}}) \preccurlyeq_{\mathrm{SC}} \bar{f}_{\mathrm{FV}}(\mathbf{x})$. Because of the disjunction in $\preccurlyeq_{\mathrm{sC}}$, the proof consists of four parts. For the purpose of the proof, we consider an omitted function $f_{a}, f_{b}$, or $f_{c}$ to be constant.

1. If $\overline{\mathbf{x}}_{u}=\hat{\mathbf{x}}_{u} \wedge f_{l}(\overline{\mathbf{x}}) \leq f_{l}(\hat{\mathbf{x}})$ and $\hat{\mathbf{x}}_{u}=\mathbf{x}_{u} \wedge f_{l}(\hat{\mathbf{x}}) \leq f_{l}(\mathbf{x})$, then $\overline{\mathbf{x}}_{u}=\mathbf{x}_{u} \wedge f_{l}(\overline{\mathbf{x}}) \leq f_{l}(\mathbf{x})$ and, hence, $\bar{f}_{\mathrm{FV}}(\overline{\mathbf{x}}) \preccurlyeq \mathrm{sC} \bar{f}_{\mathrm{FV}}(\mathbf{x})$.

2. If $\neg\left(\hat{\mathbf{x}}_{u}=\overline{\mathbf{x}}_{u} \wedge f_{l}(\hat{\mathbf{x}}) \leq f_{l}(\overline{\mathbf{x}})\right) \wedge f_{u}(\overline{\mathbf{x}}) \leq f_{u}(\hat{\mathbf{x}})$ and $\hat{\mathbf{x}}_{u}=\mathbf{x}_{u} \wedge f_{l}(\hat{\mathbf{x}}) \leq f_{l}(\mathbf{x})$, it follows that $f_{a}\left(\hat{\mathbf{x}}_{u}\right)=f_{a}\left(\mathbf{x}_{u}\right), f_{b}(\hat{\mathbf{x}}) \leqq f_{b}(\mathbf{x})$, and, therefore, $f_{u}(\hat{\mathbf{x}}) \leqq f_{u}(\mathbf{x})$.

(a) If $\hat{\mathbf{x}}_{u} \neq \overline{\mathbf{x}}_{u}$, then $\mathbf{x}_{u} \neq \overline{\mathbf{x}}_{u}$.

(b) If $\hat{\mathbf{x}}_{u}=\overline{\mathbf{x}}_{u}$, then $f_{l}(\hat{\mathbf{x}}) \not \leq f_{l}(\overline{\mathbf{x}})$ and, therefore, $f_{l}(\mathbf{x}) \not \leq f_{l}(\overline{\mathbf{x}})$.

In both cases, it follows that $\neg\left(\mathbf{x}_{u}=\overline{\mathbf{x}}_{u} \wedge f_{l}(\mathbf{x}) \leq f_{l}(\overline{\mathbf{x}})\right) \wedge f_{u}(\overline{\mathbf{x}}) \leq f_{u}(\mathbf{x})$ and, hence, $\bar{f}_{\mathrm{FV}}(\overline{\mathbf{x}}) \preccurlyeq \mathrm{SC} \bar{f}_{\mathrm{FV}}(\mathbf{x})$. 
3. If $\overline{\mathbf{x}}_{u}=\hat{\mathbf{x}}_{u} \wedge f_{l}(\overline{\mathbf{x}}) \leq f_{l}(\hat{\mathbf{x}})$ and $\neg\left(\mathbf{x}_{u}=\hat{\mathbf{x}}_{u} \wedge f_{l}(\mathbf{x}) \leq f_{l}(\hat{\mathbf{x}})\right) \wedge f_{u}(\hat{\mathbf{x}}) \leq f_{u}(\mathbf{x})$, it follows that $f_{a}\left(\overline{\mathbf{x}}_{u}\right)=f_{a}\left(\hat{\mathbf{x}}_{u}\right), f_{b}(\overline{\mathbf{x}}) \leqq f_{b}(\hat{\mathbf{x}})$, and, therefore, $f_{u}(\overline{\mathbf{x}}) \leqq f_{u}(\hat{\mathbf{x}})$.

(a) If $\mathbf{x}_{u} \neq \hat{\mathbf{x}}_{u}$, then $\mathbf{x}_{u} \neq \overline{\mathbf{x}}_{u}$.

(b) If $\mathbf{x}_{u}=\hat{\mathbf{x}}_{u}$, then $f_{l}(\mathbf{x}) \not \leq f_{l}(\hat{\mathbf{x}})$ and, therefore, $f_{l}(\mathbf{x}) \not \leq f_{l}(\overline{\mathbf{x}})$.

In both cases, it follows that $\neg\left(\mathbf{x}_{u}=\overline{\mathbf{x}}_{u} \wedge f_{l}(\mathbf{x}) \leq f_{l}(\overline{\mathbf{x}})\right) \wedge f_{u}(\overline{\mathbf{x}}) \leq f_{u}(\mathbf{x})$ and, hence, $\bar{f}_{\mathrm{FV}}(\overline{\mathbf{x}}) \preccurlyeq{ }_{\mathrm{SC}} \bar{f}_{\mathrm{FV}}(\mathbf{x})$.

4. If $\neg\left(\hat{\mathbf{x}}_{u}=\overline{\mathbf{x}}_{u} \wedge f_{l}(\hat{\mathbf{x}}) \leq f_{l}(\overline{\mathbf{x}})\right) \wedge f_{u}(\overline{\mathbf{x}}) \leq f_{u}(\hat{\mathbf{x}})$ and $\neg\left(\mathbf{x}_{u}=\hat{\mathbf{x}}_{u} \wedge f_{l}(\mathbf{x}) \leq f_{l}(\hat{\mathbf{x}})\right) \wedge f_{u}(\hat{\mathbf{x}}) \leq$ $f_{u}(\mathbf{x})$, it follows that $f_{u}(\overline{\mathbf{x}}) \leq f_{u}(\mathbf{x})$. If $\mathbf{x}_{u}=\overline{\mathbf{x}}_{u}$, which is possible only if $f_{b}$ is nonconstant, it follows that $f_{a}\left(\overline{\mathbf{x}}_{u}\right)=f_{a}\left(\mathbf{x}_{u}\right)$ and $f_{b}(\overline{\mathbf{x}}) \leq f_{b}(\mathbf{x})$, which implies that $f_{b}(\mathbf{x}) \not f_{b}(\overline{\mathbf{x}})$ and, thus, that $f_{l}(\mathbf{x}) \not \leq f_{l}(\overline{\mathbf{x}})$. Therefore, $\neg\left(\mathbf{x}_{u}=\overline{\mathbf{x}}_{u} \wedge f_{l}(\mathbf{x}) \leq f_{l}(\overline{\mathbf{x}})\right) \wedge f_{u}(\overline{\mathbf{x}}) \leq f_{u}(\mathbf{x})$ and, hence, $\bar{f}_{\mathrm{FV}}(\overline{\mathbf{x}}) \preccurlyeq_{\mathrm{SC}} \bar{f}_{\mathrm{FV}}(\mathbf{x})$, which completes the proof.

The second special case is a problem that originated from considering a multiobjective problem decomposed into a collection of subproblems each involving some subset of the original objectives $[43,44]$. The aim is to find the decisions that are efficient both with respect to all the objectives and with respect to a given subset of the objectives. That is to say, we look for those efficient decisions that would remain efficient if only the given subset of objectives were retained.

Let $X \subset \mathbb{R}^{n}$ be a nonempty set, and let $f_{a}: X \rightarrow \mathbb{R}^{p_{a}}$ and $f_{b}: X \rightarrow \mathbb{R}^{p_{b}}$ be two vectorvalued functions. The above-described problem can be written as

$$
\min \left(f_{a}, f_{b}\right)(\mathbf{x}) \text { subject to } \mathbf{x} \in E\left(X, f_{b}, \leq\right),
$$

where the function $f_{b}$ encompasses the objectives that belong to the given subset and the function $f_{a}$ the objectives that do not. At the lower level, the optimization is with respect to all decision variables, that is, $\mathbf{x}_{l}=\mathbf{x}$, and the lower-level problem is not parameterized by any upper-level decision $\mathbf{x}_{u}$. The upper-level and the lower-level objective functions are $f_{u}=\left(f_{a}, f_{b}\right)$ and $f_{l}=f_{b}$, respectively.

Because there is no upper-level decision $\mathbf{x}_{u}$ in (13), the relation $\preccurlyeq_{\mathrm{sC}}$ can be written as

$$
\begin{aligned}
\bar{f}_{\mathrm{FV}}(\overline{\mathbf{x}}) \preccurlyeq_{\mathrm{SC}} \bar{f}_{\mathrm{FV}}(\mathbf{x}) & \Longleftrightarrow f_{l}(\overline{\mathbf{x}}) \leq f_{l}(\mathbf{x}) \vee\left(f_{l}(\mathbf{x}) \not \leq f_{l}(\overline{\mathbf{x}}) \wedge f_{u}(\overline{\mathbf{x}}) \leq f_{u}(\mathbf{x})\right) \\
& \Longleftrightarrow f_{l}(\overline{\mathbf{x}}) \leq f_{l}(\mathbf{x}) \vee f_{u}(\overline{\mathbf{x}}) \leq f_{u}(\mathbf{x}) .
\end{aligned}
$$

The latter equivalence follows from the fact that $f_{u}(\overline{\mathbf{x}}) \leq f_{u}(\mathbf{x})$ implies $f_{l}(\mathbf{x}) \not \leq f_{l}(\overline{\mathbf{x}})$, which thus becomes redundant in the conjunction. The following proposition provides means to solve (13) as a MOP.

Proposition 5.2 Let the set $X$ and the functions $f_{u}$ and $f_{l}$ be defined as in (13). The efficient set $E\left(X, \bar{f}_{\mathrm{FV}}, \preccurlyeq_{\mathrm{sC}}\right)$ is equal to the efficient set $E\left(X_{\mathrm{I}}, f_{u}, \leq\right)$ of problem (13).

Proof The proof is similar to that of Proposition 5.1. By Proposition 4.1 and Corollary 4.1, it is sufficient to show that the relation $\preccurlyeq$ sc is transitive for problem (13). The simplified expression given by (14) is used. Because of the disjunction in $\preccurlyeq_{\mathrm{SC}}$, the proof consists of four parts.

1. If $f_{l}(\overline{\mathbf{x}}) \leq f_{l}(\hat{\mathbf{x}})$ and $f_{l}(\hat{\mathbf{x}}) \leq f_{l}(\mathbf{x})$, it follows that $f_{l}(\overline{\mathbf{x}}) \leq f_{l}(\mathbf{x})$ and, hence, $\bar{f}_{\mathrm{FV}}(\overline{\mathbf{x}}) \preccurlyeq \mathrm{sC}$ $\bar{f}_{\mathrm{FV}}(\mathbf{x})$.

2. If $f_{u}(\overline{\mathbf{x}}) \leq f_{u}(\hat{\mathbf{x}})$ and $f_{l}(\hat{\mathbf{x}}) \leq f_{l}(\mathbf{x})$, it follows that $f_{b}(\overline{\mathbf{x}}) \leqq f_{b}(\hat{\mathbf{x}}) \Longleftrightarrow f_{l}(\overline{\mathbf{x}}) \leqq f_{l}(\hat{\mathbf{x}})$. Thus, $f_{l}(\overline{\mathbf{x}}) \leq f_{l}(\mathbf{x})$ and, hence, $\bar{f}_{\mathrm{FV}}(\overline{\mathbf{x}}) \preccurlyeq$ sC $\bar{f}_{\mathrm{FV}}(\mathbf{x})$.

3. If $f_{l}(\overline{\mathbf{x}}) \leq f_{l}(\hat{\mathbf{x}})$ and $f_{u}(\hat{\mathbf{x}}) \leq f_{u}(\mathbf{x})$, it follows that $f_{b}(\hat{\mathbf{x}}) \leqq f_{b}(\mathbf{x}) \Longleftrightarrow f_{l}(\hat{\mathbf{x}}) \leqq f_{l}(\mathbf{x})$. Thus, $f_{l}(\overline{\mathbf{x}}) \leq f_{l}(\mathbf{x})$ and, hence, $\bar{f}_{\mathrm{FV}}(\overline{\mathbf{x}}) \preccurlyeq \mathrm{sC} \bar{f}_{\mathrm{FV}}(\mathbf{x})$. 
4. If $f_{u}(\overline{\mathbf{x}}) \leq f_{u}(\hat{\mathbf{x}})$ and $f_{u}(\hat{\mathbf{x}}) \leq f_{u}(\mathbf{x})$, it follows that $f_{u}(\overline{\mathbf{x}}) \leq f_{u}(\mathbf{x})$ and, hence, $\bar{f}_{\mathrm{FV}}(\overline{\mathbf{x}}) \preccurlyeq \mathrm{sC}$ $\bar{f}_{\mathrm{FV}}(\mathbf{x})$, which completes the proof.

The special case (13) is distinct from the special case (12) because in the latter an objective function depending on the lower-level decision $\mathbf{x}_{l}$ is not allowed to appear only at the upper level. However, with $f_{a}, f_{b}$, and $X$ as in (13), the special case (13) is equivalent to BMOP

$$
\min f_{b}(\mathbf{x}) \text { subject to } \mathbf{x} \in E\left(X,\left(f_{a}, f_{b}\right), \leq\right)
$$

which is a valid instantiation of (12). The equivalence of problems (13) and (15) is easily verified by repeating the derivation given in (14) for problem (15); the resulting relation, when written in terms of $f_{a}$ and $f_{b}$, will be identical to the relation derived in (14) and, thus, the efficient sets of (13) and (15) must be equal.

\section{Conclusion}

In this paper, we brought together theorems connecting bilevel optimization and multiobjective optimization and provided sufficient conditions for a binary relation to reduce a BMOP to a MOP. We generalized the results that assumed scalar-valued objective functions for bilevel multiobjective optimization and presented all the results in a unified form for the first time. We also proposed a simple binary relation satisfying the sufficient conditions in certain special cases. In those special cases, the proposed relation allows a BMOP to be reduced to a single-level MOP, which may result in considerable savings in the computational effort required to solve such problems. Two special cases of a general BMOP, both originally motivated by the needs of applications, were introduced and successfully reduced to MOPs. The two special cases were found to be distinct, but closely related. It remains unclear, however, whether there exists a common formulation covering both special cases and allowing a comparable reduction.

Possible directions for future research include studying the practical aspects of implementing a solution approach based on the results presented, applying existing solution methods to the multiobjective problems resulting from the reduction to learn how difficult they are to solve, and designing new algorithms for bilevel multiobjective optimization that make use of the achieved reduction.

\section{References}

1. Vicente, L.N., Calamai, P.H.: Bilevel and multilevel programming: A bibliography review. Journal of Global Optimization 5(3), 291-306 (1994)

2. Dempe, S.: Annotated bibliography on bilevel programming and mathematical programs with equilibrium constraints. Optimization 52(3), 333-359 (2003)

3. Colson, B., Marcotte, P., Savard, G.: An overview of bilevel optimization. Annals of Operations Research 153(1), 235-256 (2007)

4. Bard, J.F.: Practical Bilevel Optimization: Algorithms and Applications. Kluwer Academic Publishers (1998)

5. Dempe, S.: Foundations of Bilevel Programming. Kluwer Academic Publishers (2002)

6. Marcotte, P., Savard, G.: A note on the Pareto optimality of solutions to the linear bilevel programming problem. Computers \& Operations Research 18(4), 355-359 (1991)

7. Fülöp, J.: On the equivalency between a linear bilevel programming problem and linear optimization over the efficient set. Tech. Rep. WP 93-1, Laboratory of Operations Research and Decision Systems, Computer and Automation Institute, Hungarian Academy of Sciences (1993) 
8. Glackin, J., Ecker, J.G., Kupferschmid, M.: Solving bilevel linear programs using multiple objective linear programming. Journal of Optimization Theory and Applications 140(2), 197-212 (2009)

9. Alves, M.J., Dempe, S., Júdice, J.J.: Computing the Pareto frontier of a bi-objective bi-level linear problem using a multiobjective mixed-integer programming algorithm. Optimization (2010). doi: 10.1080/02331934.2010.511674. First published on: 13 September 2010 (iFirst)

10. Fliege, J., Vicente, L.N.: Multicriteria approach to bilevel optimization. Journal of Optimization Theory and Applications 131(2), 209-225 (2006)

11. Ivanenko, D.S., Plyasunov, A.V.: Reducibility of bilevel programming problems to vector optimization problems. Journal of Applied and Industrial Mathematics 2(2), 179-195 (2008)

12. Pieume, C.O., Fotso, L.P., Siarry, P.: Solving bilevel programming problems with multicriteria optimization techniques. OPSEARCH 46(2), 169-183 (2009)

13. Calvete, H.I., Galé, C.: Linear bilevel programs with multiple objectives at the upper level. Journal of Computational and Applied Mathematics 234(4), 950-959 (2010)

14. Nie, P.: A note on bilevel optimization problems. International Journal of Applied Mathematical Sciences 2(1), 31-38 (2005)

15. Bonnel, H., Morgan, J.: Semivectorial bilevel optimization problem: Penalty approach. Journal of Optimization Theory and Applications 131(3), 365-382 (2006)

16. Nishizaki, I., Sakawa, M.: Stackelberg solutions to multiobjective two-level linear programming problems. Journal of Optimization Theory and Applications 103(1), 161-182 (1999)

17. Sakawa, M., Nishizaki, I.: Cooperative and Noncooperative Multi-Level Programming. Springer (2009)

18. Dell'Aere, A.: Numerical methods for the solution of bi-level multi-objective optimization problems. Ph.D. thesis, Universität Paderborn (2008)

19. Arora, S.R., Arora, R.: Indefinite quadratic bilevel programming problem with multiple objectives at both levels. International Journal of Optimization: Theory, Methods and Applications 1(3), 318-327 (2009)

20. Eichfelder, G.: Multiobjective bilevel optimization. Mathematical Programming 123(2), 419-449 (2010)

21. Gebhardt, E., Jahn, J.: Global solver for nonlinear bilevel vector optimization problems. Pacific Journal of Optimization 5(3), 387-401 (2009)

22. Shi, X., Xia, H.: Interactive bilevel multi-objective decision making. Journal of the Operational Research Society 48(9), 943-949 (1997)

23. Shi, X., Xia, H.S.: Model and interactive algorithm of bi-level multi-objective decision-making with multiple interconnected decision makers. Journal of Multi-Criteria Decision Analysis 10(1), 27-34 (2001)

24. Abo-Sinna, M.A., Baky, I.A.: Interactive balance space approach for solving multi-level multi-objective programming problems. Information Sciences 117(16), 3397-3410 (2007)

25. Osman, M.S., Abo-Sinna, M.A., Amer, A.H., Emam, O.E.: A multi-level non-linear multi-objective decision-making under fuzziness. Applied Mathematics and Computation 153(1), 239-252 (2004)

26. Zhang, G., Lu, J., Dillon, T.: Solution concepts and an approximation Kuhn-Tucker approach for fuzzy multiobjective linear bilevel programming. In: A. Chinchuluun, P.M. Pardalos, A. Migdalas, L. Pitsoulis (eds.) Pareto Optimality, Game Theory and Equilibria, pp. 457-480. Springer (2008)

27. Yano, H., Sakawa, M.: A fuzzy approach to hierarchical multiobjective programming problems and its application to an industrial pollution control problem. Fuzzy Sets and Systems 160(22), 3309-3322 (2009)

28. Baky, I.A.: Fuzzy goal programming algorithm for solving decentralized bi-level multi-objective programming problems. Fuzzy Sets and Systems 160(18), 2701-2713 (2009)

29. Baky, I.A.: Solving multi-level multi-objective linear programming problems through fuzzy goal programming approach. Applied Mathematical Modelling 34(9), 2377-2387 (2010)

30. Yin, Y.: Multiobjective bilevel optimization for transportation planning and management problems. Journal of Advanced Transportation 36(1), 93-105 (2002)

31. Deb, K., Sinha, A.: Solving bilevel multi-objective optimization problems using evolutionary algorithms. In: M. Ehrgott, C. Fonseca, X. Gandibleux, J.K. Hao, M. Sevaux (eds.) Evolutionary Multi-Criterion Optimization: 5th International Conference; Proceedings, pp. 110-124. Springer (2009)

32. Jia, L., Wang, Y.: A genetic algorithm for multiobjective bilevel convex optimization problems. In: Proceedings of International Conference on Computational Intelligence and Security, pp. 98-102. IEEE Computer Society (2009)

33. Ehrgott, M.: Multicriteria Optimization, second edn. Springer (2005)

34. Jahn, J.: Vector Optimization: Theory, Applications, and Extensions. Springer (2004)

35. Miettinen, K.: Nonlinear Multiobjective Optimization. Kluwer Academic Publishers (1999)

36. Philip, J.: Algorithms for the vector maximization problem. Mathematical Programming 2(1), 207-229 (1972)

37. Benson, H.P.: An all-linear programming relaxation algorithm for optimizing over the efficient set. Journal of Global Optimization 1(1), 83-104 (1991) 
38. Benson, H.P.: A finite, nonadjacent extreme-point search algorithm for optimization over the efficient set. Journal of Optimization Theory and Applications 73(1), 47-64 (1992)

39. Ecker, J.G., Song, J.H.: Optimizing a linear function over an efficient set. Journal of Optimization Theory and Applications 83(3), 541-563 (1994)

40. Sayin, S.: Optimizing over the efficient set using a top-down search of faces. Operations Research 48(1), 65-72 (2000)

41. Yamamoto, Y.: Optimization over the efficient set: overview. Journal of Global Optimization 22(1-4), 285-317 (2002)

42. Ropponen, A., Ritala, R., Pistikopoulos, E.N.: Optimization issues of the broke management system in papermaking. Computers and Chemical Engineering (2010). doi: 10.1016/j.compchemeng.2010.12.012

43. Eskelinen, P., Ruuska, S., Miettinen, K., Wiecek, M.M., Mustajoki, J.: A scenario-based interactive multiobjective optimization method for decision making under uncertainty. In: C.H. Antunes, D.R. Insua, L.C. Dias (eds.) Proceedings of the 25th Mini-EURO Conference on Uncertainty and Robustness in Planning and Decision Making (URPDM2010) (2010)

44. Engau, A., Wiecek, M.M.: Interactive coordination of objective decompositions in multiobjective programming. Management Science 54(7), 1350-1363 (2008) 